\title{
Functional Determinants in Euclidean Yang-Mills Theory $\star$
}

\author{
Constantine Callias ${ }^{1}$ and Clifford $\mathrm{H}$. Taubes ${ }^{2}$ \\ ${ }^{1}$ Center for Theoretical Physics, Laboratory for Nuclear Science and Department of Physics, \\ Massachusetts Institute of Technology, Cambridge, MA, 02139, USA \\ ${ }^{2}$ Lyman Laboratory of Physics, Harvard University, Cambridge, MA, 02136, USA
}

\begin{abstract}
We study functional determinants entering the path integral for topologically non-trivial sectors of Euclidean SU(2) Yang-Mills theory and we derive some results in exact closed form.
\end{abstract}

\section{Introduction}

We present in this paper a calculation in exact closed form of some functional determinants arising in the study of quantum corrections about multi-pseudoparticle solutions to Euclidean SU(2) Yang-Mills theory.

Our calculation is a continuation of the results of 't Hooft [1] on the quantum field theory about the single pseudoparticle solution. The isospin- $1 / 2$ and fermion determinants that we calculate, however, only partially extend this program.

We have not attempted in this paper to deal with the general $8 n-3$ parameter multi-pseudoparticle solutions. Rather we restrict ourselves to the $5 n$-parameter one [2]. The obvious extension to the $5 n+4$ parameters [2] is impossible for technical reasons having to do with the asymptotic behavior at spatial infinity, as will become apparent in the statement of Proposition 5. Other authors [3] however, have studied the properties of the determinant under conformal transformations and the results can be used to pass from the $5 n$ to the $5 n+4$ parameters. The case we are studying here has also been attacked independently by Brown and Creamer [4] who use a point-splitting method of regularization and previous knowledge about the Green's function for the covariant Laplacian to calculate the determinant.

Our method uses a Pauli-Villars type of regularization, and the derivation although truly simple covers a much wider class of background configurations than just the self-dual solutions to the Yang-Mills equations; in fact it includes an infinite dimensional manifold of non-self-dual backgrounds. The final answer can

* This work is supported through funds provided by the US Department of Energy (DOE) under Contract EY-76-C-02-3069 and by the National Science Foundation through funds provided under Contract PHY 79-16812 
be exhibited in the form of a four-dimensional integral when use is made of the theory of the asymptotics of the kernel function for the heat equation.

We shall see, however, in Sect. 3 and Appendix A that for background potentials of non-trivial Pontryagin number, the operators involved in these heat equations are differential operators with singular coefficients. The ordinary asymptotic theory does not apply in this case. Unfortunately we have not been able to find a direct way of systematically studying the asymptotics. In our calculation, however, we only need a few terms in the expansion, and, as it turns out, those can be obtained by approximating the multi-instanton potential by the single instanton one at the singular points. These restricted results show clearly that the small $s$ behavior of $e^{-s H}(x, x)$, where $H$ is a differential operator with singular coefficients, contains terms of a kind different from the ordinary power series. We formulate a general conjecture that extends our low order calculations.

In Sect. 2 we give a definition of the regularized determinant and we describe the differential operators involved. In Sect. 3 we derive a formula for the determinant, using results on the heat kernels derived in Appendix A. The first two appendices provide as much of the theory of the heat equation as is needed in the paper.

\section{Definition of the Determinant}

\section{a) Description of the Operators}

It is our goal to compute the determinants of the operators $H^{2}$ and $D^{\dagger} D$, where

$$
H=\left(\begin{array}{ll}
0 & D \\
D^{\dagger} & 0
\end{array}\right),
$$

where $D$ is a first order differential operator to be defined below. We shall soon see that each of these determinants can be obtained from the other and thus we shall compute the determinant of $D^{\dagger} D$ in the next Section.

The operator $H$ above represents the Dirac operator in Euclidean four-space in the background of SU(2) instantons [5] and $D^{\dagger} D$ turns out to be the operator of scalar fields of isospin-1/2 in the same background. Precisely, $D$ is given by [5]

$$
D=i \alpha^{\mu} \otimes\left(\mathbb{1} \cdot \partial_{\mu}+A_{\mu}\right),
$$

where

$$
A_{\mu}=i \bar{\tau}^{\mu \nu} \partial_{\nu} \ln \varrho
$$

is the vector potential of the $n$-instanton solution of 't Hooft [2] in a convenient representation of the gauge fields. The tensor product appearing in the operator $D$ represents a tensor product of vector spaces. The four matrices $\alpha^{\mu}$ are $2 \times 2$ matrices defined by the algebra they satisfy (here $\bar{\alpha}^{\mu}=\alpha^{\mu \dagger}$ )

$$
\begin{aligned}
& \bar{\alpha}^{\mu} \alpha^{v}+\bar{\alpha}^{v} \alpha^{\mu}=\delta^{\mu \nu} \\
& \alpha^{\mu} \bar{\alpha}^{v}+\alpha^{v} \bar{\alpha}^{\mu}=\delta^{\mu \nu} .
\end{aligned}
$$


A possible representation for them is $\alpha^{0}=1, \alpha^{k}=i \sigma^{k}$, where $\sigma^{k}$ are the Pauli matrices. The $\bar{\tau}^{\mu \nu}$ appearing in the definition of $A_{\mu}$ are defined in terms of matrices $\beta^{\mu}$, that satisfy the same algebra as the $\alpha^{\mu}$, as follows

$$
\bar{\tau}^{\mu v}=\frac{1}{4 i}\left(\beta^{\mu} \bar{\beta}^{v}-\beta^{v} \bar{\beta}^{\mu}\right) .
$$

Finally the function $\varrho$ in the formula for $A_{\mu}$ is given by [2]

$$
\varrho(x)=1+\sum_{k=1}^{n} \frac{\lambda_{k}^{2}}{\left(x-y_{k}\right)^{2}} \quad \lambda_{k} \in \mathbb{R}, y_{k} \in \mathbb{R}^{4} .
$$

This function is a solution of the following equation on $\mathbb{R}^{4}$ :

$$
1 / \varrho \partial_{\mu} \partial_{\mu} \varrho=0
$$

which is the necessary and sufficient condition for the gauge field

$$
F_{\mu \nu}=\partial_{\mu} A_{v}-\partial_{v} A_{\mu}+\left[A_{\mu}, A_{v}\right]
$$

derived from a gauge potential of the form (2.3) to be self-dual, i.e., to satisfy

$$
F_{\mu \nu}={ }^{*} F_{\mu \nu} \equiv \frac{1}{2} \varepsilon^{\mu \nu \alpha \beta} F_{\alpha \beta}
$$

$\left(\varepsilon_{0123}=1\right)$.

It can be shown easily [5] that if the condition of self-duality is satisfied,

$$
D^{\dagger} D=D_{\mu} D_{\mu} \oplus D_{\mu} D_{\mu},
$$

where

$$
D_{\mu}=\mathbb{1} \cdot \partial_{\mu}+A_{\mu}
$$

is the operator of the covariant derivative for isospin- $1 / 2$ scalar fields in the background of the gauge potential $A_{\mu}$.

\section{b) The Determinant of Second Order Differential Operators on $\mathbb{R}^{m}$}

We now proceed to define the determinant of a self-adjoint positive differential operator. We first consider the case where the base manifold is compact. Let $M$ be a compact orientable manifold and $E$ a real $C^{\infty}$ vector bundle with $C^{\infty}$ scalar product defined over $M$. Define $\Gamma(E)$ to be the set of $C^{\infty}$ cross-sections of $E$. Let $A$ be a second order positive operator which is essentially self-adjoint on the dense domain $\Gamma(E)$. We denote by $A$ also the Friedrich's extension of $A$. As is well known, naive definitions of such quantities as operator determinants on compact manifolds can contain so-called ultraviolet divergences corresponding to the short distance singularities of the kernels of the differential operators. We choose to treat these within the scheme of Pauli-Villars regulators. Suppose we are interested in computing the determinant of a second order differential operator with dense domain $\Gamma(E)$ and we know the determinant of $A_{0}$, another second order, positive self-adjoint, differential operator with the same dense domain. Notice that if $A$ and 
$A_{0}$ were finite dimensional square matrices with the same rank, the ratio of their determinants is given by

$$
\ln \operatorname{det} A A_{0}^{-1}=-\int_{0}^{\infty} \frac{d s}{s} \operatorname{Tr}\left(e^{-s A}-e^{-s A_{0}}\right) .
$$

We shall extend this definition to second order positive self-adjoint operators with identical cores, $\Gamma(E)$. The operator $e^{-s A}$ is in this case an integral operator with kernel in local coordinates $e^{-s A}(x, y)$ and

$$
\operatorname{tr} e^{-s A}=\int_{M}\left[\operatorname{Tr} e^{-s A}(x, x)\right] d w(x),
$$

where $d w(x)$ is the natural volume form on $M$ and tr stands for the trace over the matrix indices as defined by the scalar product on $E$.

The ultraviolet problem manifests itself in (2.12) in the small $s$ behavior of the integrand. In fact, for a second order operator with $C^{\infty}$ coefficients on a manifold of dimension $m$ we have asymptotically

$$
e^{-s A}(x, x) \sim a_{-\frac{m}{2}}(x) \frac{1}{s^{m / 2}}+a_{-\frac{m}{2}+1}(x) \frac{1}{s^{m / 2-1}}+\ldots
$$

which implies, in our situation,

$$
e^{-s A}(x, x)-e^{-s A_{0}}(x, x) \sim a_{-1}(x) \frac{1}{s}+a_{0}(x)+\ldots .
$$

As we shall see in the following section, if the coefficients of $A$ are singular at a finite number of points, this asymptotic expansion may contain logarithmic terms as well, but nothing more singular than what is included here.

To remove the $s \rightarrow 0$ singularities we introduce a regularizing factor $r(s)$ in the integral (2.12) defined by

$$
r(s)=\sum_{i=0}^{R} e_{i} e^{-s z_{i}},
$$

where $e_{i}=(-1)^{i}, R$ is odd, $z_{0}=0$ and the $z_{i}$ satisfy

$$
\begin{aligned}
& \sum_{i=1}^{R} e_{i} z_{i}=0 \\
& \sum_{i=1}^{R} e_{i} z_{i}^{2}=0 \\
& \vdots \\
& \sum_{i=1}^{R} e_{i} z_{i}^{k} \ln z_{i}=-z_{(k)}^{k} \ln z_{(k)}, \quad k=0, \ldots, \frac{m}{2}-1 .
\end{aligned}
$$

At the end of the calculation we take the limit $z_{i} \rightarrow \infty, i \geqq 1$ keeping these conditions as constraints and $z_{(k)}$ constant. The insertion (2.16) amounts to a PauliVillars regulation [6] of the determinant. 
We summarize the above discussion in

Definition 1. The Pauli-Villars regularized ratio of determinants of operators $A$ and $A_{0}$ with the same dense domains, $\Gamma(E)$ (the set of $C^{\infty}$ cross-sections of a $C^{\infty}$ vector bundle $E$ over $M$ ) is defined by

$$
\ln \left(\operatorname{det} A / \operatorname{det} A_{0}\right)=-\lim _{z_{1}, \ldots, z_{R} \rightarrow \infty} \int_{0}^{\infty} \frac{d s}{s} \operatorname{Tr}\left(e^{-s A}-e^{-s A_{0}}-\Pi+\Pi_{0}\right) r(s),
$$

where $r(s)$ is given by (2.16) and the $z_{i}$ satisfy relation (2.17). $\Pi$ and $\Pi_{0}$ are the projections onto the eigenspaces of the eigenvalue zero of the operators $A$ and $A_{0}$, respectively.

The only ingredient in this definition not included in the above discussion is the subtraction of the zero-eigenspace from the trace. This is done to avoid trivial vanishing of the determinant due to zero eigenvalues.

We now consider the case of interest where the base manifold is non-compact and diffeomorphic to $\mathbb{R}^{n}$. In this case all vector bundles are isomorphic to the trivial bundle and hence can be represented as the direct sum of $m$ copies of $\mathbb{R}$ where $m$ is the dimensions of the vector bundle. There exists a natural choice for the operator $A_{0}$ in this case:

$$
A_{0}=\Delta_{0} \mathbb{1} \text {, }
$$

where $\Delta_{0}$ is the scalar Laplace operator on $M$ and $\mathbb{1}$ is the identity automorphism of $\mathbb{R}^{m}$.

On a non-compact manifold an operator expression such as (2.13) may diverge due to the infinite volume of the manifold. The expression (2.18) for the ratio of the determinants of $A$ and $A_{0}$ in general requires additional regularization in order to be well defined. From now on we take $M=\mathbb{R}^{n}$. If $A$ has the second order term $-\partial_{\mu} \partial_{\mu} \cdot \mathbb{1}$, then $e^{-s A}(x, x)$ does not go to zero as $|x| \rightarrow \infty$.

$$
\lim _{|x| \rightarrow \infty} e^{-s A}(x, x)=\frac{1}{16 \pi^{2}} \frac{1}{s^{2}} \mathbb{1},
$$

This, however, is just $e^{-s A_{0}}(x, x)$ with $A_{0}$ given by (2.19). This choice of $A_{0}$ corrects part of the infrared problem. What remains may still not approach zero as $|x| \rightarrow \infty$ fast enough to be integrable. Actually, as we shall see, this appears to be the case in our problem but the slowly decaying terms cancel. To make the cancellation unambiguous we insert a function, $\chi_{\lambda}(x), \lambda>0$, into the integral defining the trace in (2.18) with the properties

(a) $\chi_{\lambda}(x)=1$ for $|x| \leqq \lambda$,

(b) $\chi_{\lambda}(x)=0$ for $|x| \geqq \lambda+1$,

(c) $\chi_{\lambda}(x)$ is infinitely differentiable.

We then define the ratio of determinants as the limit as $\lambda \rightarrow \infty$.

We summarize the above discussion for the case $M=\mathbb{R}^{n}$ in

Definition 2. Let $E=\mathbb{R}^{m}$ be an $m$ dimensional vector bundle over $\mathbb{R}^{n}$. The PauliVillars regularized determinant of a positive, self-adjoint, differential operator $A=-\partial_{\mu} \partial_{\mu} \cdot \mathbb{1}+\left(\right.$ lower order terms) with dense domain $\left[C_{0}^{\infty}\left(\mathbb{R}^{n}\right)\right]^{m}$ is defined by

$$
\left.\ln \operatorname{det} A=-\lim _{z_{1}, \ldots, z_{R} \rightarrow \infty} \lim _{\lambda \rightarrow \infty} \int_{0}^{\infty} \frac{d s}{s} \operatorname{Tr} \chi_{\lambda}\left(e^{-s A}-e^{-s\left(-\hat{\partial}_{\mu} \partial_{\mu}\right.}\right)-\Pi\right) r(s),
$$


where $r(s)$ is given by (2.16) and the $z_{i}$ satisfy the relation (2.17). $\Pi$ is the projection onto the eigenspace of the eigenvalue zero of the operator $A . \chi_{\lambda}, \lambda>0$ is defined by properties (a), (b), and (c) above.

From the definition we can study how the determinant is affected by this regularization. Proposition 1 tells us that the determinant is gauge invariant. Proposition 2 is stated for dimension 4 but it can easily be generalized to other dimensions.

Proposition 1. Let $A, \tilde{A}, A_{0}=-\partial^{2} 1$ be as in Definition 2 above and suppose $\tilde{A}$ is locally unitarily similar to $A$, i.e. $\tilde{A}=g(x) A g(x)^{-1}, g(x)$ a unitary transformation of $\mathbb{R}^{m}$. Then $\operatorname{det} A=\operatorname{det} \tilde{A}$.

Proof.

$$
\begin{aligned}
\operatorname{tr} \chi_{\lambda}\left(e^{-s A}-e^{-s A_{0}}\right) & =\operatorname{tr} g \chi_{\lambda}\left(e^{-s A}-e^{-s A_{0}}\right) g^{-1}=\operatorname{tr} \chi_{\lambda}\left(g e^{-s A} g^{-1}-e^{-s A_{0}} g g^{-1}\right) \\
& =\operatorname{tr} \chi_{\lambda}\left(e^{-s g A g^{-1}}-e^{-s A_{0}}\right)
\end{aligned}
$$

since $e^{-s A_{0}}(x, x)$ is proportional to the identity matrix and therefore $g$ commutes with it.

Proposition 2. Suppose $A=-\partial^{2} \mathbb{1}+\mathbb{V}_{1}^{\mu} \cdot \partial_{\mu}+\mathbb{V}_{0}^{\mu}$ is as in Definition 2 above with $n=4$ and $\mathbb{V}_{1}^{\mu}$ and $\mathbb{V}_{0} m \times m$ matrices of smooth functions on $\mathbb{R}^{4}$. Then

(a) The kernel function $e^{-s A}(x, y)$ evaluated at $x=y$ has an asymptotic expansion of the form

$$
e^{-s A}(x, x) \sim \sum_{k=-2}^{\infty} a_{k}(x) s^{k},
$$

where $a_{-2}=$ constant, independent of $\mathbb{V}_{1}^{\mu}$ and $\mathbb{V}_{0}$, and $a_{k}(x)$ are polynomial functions of $\mathbb{V}_{1}^{\mu}(x), \mathbb{V}_{0}(x)$ and their derivatives.

(b) The determinant of $A$ can be written

$$
\ln \operatorname{det} A=\lim _{z_{i} \rightarrow \infty} \lim _{\lambda \rightarrow \infty}\left[\alpha+\beta \sum_{i=1}^{R} e_{i} \ln z_{i}+\delta \sum_{i=1}^{R} e_{i} z_{i} \ln z_{i}\right] \text {, }
$$

where

$$
\begin{aligned}
\beta= & \int_{\mathbb{R}^{4}} a_{0}(x) d x \\
\delta= & -\int_{\mathbb{R}^{4}} a_{1}(x) d x \\
\alpha= & -\int_{0}^{\infty} d s\left[\ln s \frac{\partial^{2}}{\partial s^{2}}\left(s \operatorname{Tr}\left(e^{-s A}-e^{-s A_{0}}\right)\right)\right. \\
& \left.\quad-(1+\gamma) \delta(s=0) \frac{\partial}{\partial s}\left(s \operatorname{Tr}\left(e^{-s A}-e^{-s A_{0}}\right)\right)\right],
\end{aligned}
$$

where $\gamma$ is Euler's constant, $\gamma=0.577 \ldots$

Proof. Part (a) is explained in the appendix. For part (b) one only has to integrate by parts in the formula for the determinant. The asymptotic coefficients $a_{0}$ and $a_{-1}$ are obtained when the limit $z_{i} \rightarrow \infty(i \geqq 1)$ is taken. 
As an example, we now return to the case where the positive self-adjoint operator whose determinant we are to compute is given by expressions (2.10) and (2.11). That is to say the second-order operator $-D_{v} D_{v}$ is the covariant Laplacian associated with a connection on the two complex dimensional vector bundle with structure group $S U(2)$ and base space $\mathbb{R}^{4}$. We note that in this respect we are considering the instanton connections as self-dual connections on a trivial bundle with base space $\mathbb{R}^{4}$. In Appendix $A$ we derive the coefficients $\beta$ and $\gamma$ for the covariant Laplacian $D_{v} D_{v}$. The calculation of the coefficient $\alpha$ in (2.24) is in general extremely difficult. We state the results in

Proposition 3. Suppose that $A=-D_{v} D_{v}$, the covariant Laplacian associated with a Yang-Mill's connection on the two-complex dimensional vector bundle with structure group SU(2) and base space $\mathbb{R}^{4}$. Suppose further that the connection is smooth. Then the determinant of $A$ is given as in (b) of Proposition 2, where

$$
\begin{aligned}
& \gamma=0 \\
& \text { and } \\
& \qquad \beta=\frac{1}{16 \pi^{2}} \frac{1}{12} \int d^{4} x \operatorname{tr}\left(F^{\mu v} F_{\mu \nu}\right) .
\end{aligned}
$$

\section{c) Relation of Fermion to Scalar Determinant}

Starting from our definition of the determinant above we shall show how the determinant of the square of the operator (2.1) is related to that of $D^{\dagger} D$. We shall show that

$$
\operatorname{det} H^{2}=\left(\operatorname{det} D^{\dagger} D\right)^{2}
$$

a result which can be anticipated by a formal argument. The result is proved for single instanton background fields by 't Hooft [1]. It was also derived for general background self-dual field by Brown and Creamer [4]. Here we prove it as a much more general operator identity, depending only on the decay of the coefficients of $D$ at infinity. To prove (2.27) we note that it follows from Definition 2 that

$$
\operatorname{det} H^{2}=\left(\operatorname{det} D^{\dagger} D\right) \cdot\left(\operatorname{det} D D^{\dagger}\right)
$$

and to determine $\operatorname{det} D D^{\dagger}$ in terms of $\operatorname{det} D^{\dagger} D$ note that

$$
\ln \operatorname{det} D D^{\dagger}-\ln \operatorname{det} D^{\dagger} D=-\lim _{z_{1}, \ldots, z_{R} \rightarrow \infty} \int_{0}^{s} \frac{d s}{s} r(s) \operatorname{Tr}\left(e^{-s D D^{\dagger}}-e^{-s D^{\dagger} D}-\Pi_{+}+\Pi_{-}\right) \text {, }
$$

where $\Pi_{+}\left(\Pi_{-}\right)$is the projection onto the zero-eigenvalue eigenspace of the operator $D D^{\dagger}\left(D^{\dagger} D\right)$. If we were dealing with operators on a compact manifold it would follow immediately that the trace in the integrand of the right-hand side is zero. In Appendix B we explain how things can be drastically different in the case of an open manifold due to the presence of continuous spectra. But we also show there how open space trace formulas arising in the study of the index of elliptic operators can be used to show that again

$$
\operatorname{Tr}\left(e^{-s D D^{\dagger}}-e^{-s D^{\dagger} D}\right)=\operatorname{Tr}\left(\Pi_{+}-\Pi_{-}\right)
$$

for all $s$. This proves (2.27). 


\section{Calculation of the Scalar Determinant}

As was stated in Sect. 2, the coefficient $\alpha$ in the expression (2.24) for the determinant of a second order, positive self-adjoint differential operator $A$ as described in Proposition 2 is in general extremely difficult to calculate in closed form. We prove in this section that for a certain class of operators the full determinant can, in fact, be easily calculated. It will be shown that the covariant Laplace operators associated to the $5 n$ parameter family of self-dual SU(2) connections discovered by 't Hooft [1] are in this class and we will use these operators as concrete examples.

We consider operators of the form

$$
\begin{aligned}
& H=L^{\dagger} L, \\
& L=e^{-1 / 2 \ln \varrho} i \bar{\alpha}^{\mu} \partial_{\mu} e^{1 / 2 \ln \varrho} .
\end{aligned}
$$

With $\varrho(x)$ given by (2.6), expressions (3.1) and (3.2) give the covariant Laplace operators associated with the SU(2) instantons of 't Hooft. (This fact was discovered by Brown et al. [7].) In general we will consider functions $\varrho(x)$ defined such that the 1-parameter family of operators

$$
L_{\varepsilon}=\varrho^{-\varepsilon / 2} L_{0} \varrho^{\varepsilon / 2} ; \quad 0 \leqq \varepsilon<1
$$

are closed operators on the domain $H^{1}\left(\mathbb{R}^{4}\right) \otimes \mathbb{C}^{2}$.

Lemma 1. Let $L_{\varepsilon}$ be defined by Eq. (3.3). The one parameter family of operators $L_{\varepsilon}$ are closed on $H^{1}\left(\mathbb{R}^{4}\right) \otimes \mathbb{C}^{2}$ iff $\varrho(x)$ satisfies for some $a<1, \quad b \geqq 0$ and all $\phi \in H^{1}\left(\mathbb{R}^{4}\right) \otimes \mathbb{C}^{2}$

$$
\frac{\varepsilon 2}{4} \int_{\mathbb{R}^{4}} \frac{1}{\varrho^{2}} \partial_{\mu} \varrho \partial_{\mu} \varrho \bar{\phi}^{a} \phi^{a}<\int_{\mathbb{R}^{4}}\left(a \partial_{\mu} \bar{\phi}^{a} \partial_{\mu} \phi^{a}+b \bar{\phi}^{a} \phi^{a}\right) .
$$

Proof. The operator $L_{0}=i \bar{\alpha}^{\mu} \partial_{\mu}$ is an elliptic operator that is closed on $H^{1}\left(\mathbb{R}^{4}\right) \otimes \mathbb{C}^{2}$. The operator $L_{\varepsilon}$ is of the form $L_{0}+A_{\varepsilon}$ with $A_{\varepsilon}$ a multiplication operator by a matrix function $A_{\varepsilon}(x)$. Now we use the perturbation theorem [8] that if $D\left(A_{\varepsilon}\right) \supset D\left(L_{0}\right), L_{0}$ is closed and

$$
\left\|A_{\varepsilon} \phi\right\|_{L_{2}}^{2}<a\left\|L_{0} \phi\right\|_{L_{2}}^{2}+b\|\phi\|_{L_{2}}^{2}
$$

for some $a<1, b \geqq 0$ and all $\phi \in D\left(L_{0}\right)$ then $L_{0}+A_{\varepsilon}$ is closed. QED

By a well known theorem of functional analysis, if $L_{\varepsilon}$ is as in Lemma $1, L_{\varepsilon}^{+} L_{\varepsilon}$ and $L_{\varepsilon} L_{\varepsilon}^{+}$are self-adjoint on maximal domains of definition.

For the instanton operator, with $\varrho(x)$ given by (2.6), estimate (3.4) is only satisfied for $\varepsilon^{2}<1$, for $A_{\varepsilon}(x)$ is bounded away from a finite set of points (the positions of the instantons), where it behaves like

$$
\lambda \cdot \varepsilon \frac{1}{\left|x-y_{k}\right|^{2}} \bar{\alpha}^{\mu}\left(x-y_{k}\right)_{\mu}
$$

by (2.6) and (3.4); this expression squared is just

$$
\varepsilon^{2} \frac{4}{\left|x-y_{k}\right|^{2}}
$$

and the uncertainty principle [9] in four dimensions tells us that

$$
\int d^{4} x \frac{1}{x^{2}}|\Phi|^{2} \leqq \int d^{4} x(\nabla \phi)^{2}
$$


so that if $\varepsilon^{2}<1(3.5)$ is satisfied. But notice that even though $\varepsilon=1$ is not included in the range above, the operator (3.2)

$$
H=L_{1}^{+} L_{1}
$$

can also be defined as a self-adjoint operator on a suitable domain. The reason is simply that there exist a unitary gauge transformation $g$ such that [7]

$$
g^{-1} \mathrm{Hg}
$$

is a second order differential operator of the form $-\partial^{2}+\ldots$ where the coefficients of the lower order terms are continuous functions.

In the derivation to follow one will need the self-adjointness of both $L_{\varepsilon}^{\dagger} L_{\varepsilon}$ and $L_{\varepsilon} L_{\varepsilon}^{\dagger}$, so we note first that for $\varrho(x)$ given by (2.6)

$$
\operatorname{det} L_{1}^{\dagger} L_{1}=\lim _{\varepsilon \rightarrow 1} \operatorname{det} L_{\varepsilon}^{\dagger} L_{\varepsilon}
$$

and we only need to obtain a formula for $\operatorname{det} L_{\varepsilon}^{\dagger} L_{\varepsilon}$ as a function of $\varepsilon$.

We now proceed to compute the determinant of the operator $H$ defined by (3.1) and (3.2). We first consider the case where $\varrho(x)$ is such that both $L_{\varepsilon}$ and $L_{\varepsilon}^{\dagger}$ have smooth coefficients and

$$
|\ln \varrho(x)| \leqq c \frac{1}{|x|^{2}} \quad \text { as } \quad|x| \rightarrow \infty .
$$

The conditions for Lemma 1 are satisfied here.

To make the triviality of the determinant manifest we differentiate with respect to $\varepsilon$ and show that, although we do not get zero, we obtain an exactly calculable quantity. The following manipulations on the traces will be done formally but they can easily be justified by a Levy expansion of the heat operators, of the type described in the appendix.

Note first that

$$
\begin{gathered}
\frac{\partial}{\partial \varepsilon} L_{\varepsilon}=-1 / 2\left[\ln \varrho, L_{\varepsilon}\right] \\
\frac{\partial}{\partial \varepsilon} L_{\varepsilon}^{\dagger} L_{\varepsilon}=\frac{1}{2}\left[\ln \varrho, L_{\varepsilon}^{\dagger}\right] L_{\varepsilon}-\frac{1}{2} L_{\varepsilon}^{\dagger}\left[\ln \varrho, L_{\varepsilon}\right] .
\end{gathered}
$$

Now we use the definition of the previous section for the determinant, where we note that $\Pi_{0}=0$ in our case.

Let $\operatorname{det}_{\left\{z_{z}\right\}} A$ denote the expression in Definition 1 before the cutoffs are taken to infinity. We have

$$
\begin{aligned}
\frac{\partial}{\partial \varepsilon} \ln \operatorname{det}_{\left\{z_{i}\right\}} L_{\varepsilon}^{\dagger} L_{\varepsilon}= & -\int_{0}^{\infty} \frac{d s}{s}(-s) \sum_{i=0}^{R} e_{i} e^{-s z_{i}} \\
& \cdot \operatorname{Tr}\left[\left(\left[\frac{1}{2} \ln \varrho, L_{\varepsilon}^{\dagger}\right] L_{\varepsilon} e^{-s L_{\varepsilon}^{\dagger} L_{\varepsilon}}-L_{\varepsilon}^{\dagger}\left[\frac{1}{2} \ln \varrho, L_{\varepsilon}\right] e^{-s L_{\varepsilon}^{\dagger} L_{\varepsilon}}\right) \chi_{\lambda}\right] \\
= & -\int_{0}^{\infty} d s \sum_{i=0}^{R} e_{i} e^{-s z_{1}} \operatorname{Tr}\left[\left\{\ln \varrho L_{\varepsilon}^{\dagger} L_{\varepsilon} e^{-s L_{\varepsilon}^{\dagger} L_{\varepsilon}}-\ln \varrho L_{\varepsilon}^{\dagger} e^{-s L_{\varepsilon}^{\dagger} L_{\varepsilon}} L_{\varepsilon}^{\dagger}\right\} \chi_{\lambda}\right] \\
& +O(1 / \lambda)
\end{aligned}
$$


where $\chi_{\lambda}(x)$ is defined as in Definition 2 with $\lambda>0$. The $s$-integration can be done yielding

$$
\begin{aligned}
\frac{\partial}{\partial \varepsilon} \ln \operatorname{det}_{\left\{z_{i}\right\}} L_{\varepsilon}^{\dagger} L_{\varepsilon}= & 2 \operatorname{Tr}\left[\left\{\sum_{i=0}^{R} e_{i} \frac{1}{2} \ln \varrho\left(L_{\varepsilon}^{\dagger} L_{\varepsilon} \frac{1}{L_{\varepsilon}^{\dagger} L_{\varepsilon}+z_{i}}-L_{\varepsilon} \frac{1}{L_{\varepsilon}^{\dagger} L_{\varepsilon}+z_{i}} L_{\varepsilon}^{\dagger}\right)\right\} \chi_{\lambda}\right] \\
& +O(1 / \lambda) .
\end{aligned}
$$

It is easy to see that

$$
L_{\varepsilon} \frac{1}{L_{\varepsilon}^{\dagger} L_{\varepsilon}+z_{i}}=\frac{1}{L_{\varepsilon} L_{\varepsilon}^{\dagger}+z_{i}} L_{\varepsilon}
$$

so that we obtain finally

$$
\begin{aligned}
\frac{\partial}{\partial \varepsilon} \ln \operatorname{det}_{\left\{z_{i}\right\}} L_{\varepsilon}^{\dagger} L_{\varepsilon}= & 2 \operatorname{Tr}\left[\left\{\sum_{i=0}^{R} e_{i} z_{i} \frac{1}{2} \ln \varrho\left(\frac{1}{L_{\varepsilon}^{\dagger} L_{\varepsilon}+z_{i}}-\frac{1}{L_{\varepsilon} L_{\varepsilon}^{\dagger}+z_{i}}\right)\right\} \chi_{\lambda}\right] \\
& +O(1 / \lambda) .
\end{aligned}
$$

It is convenient to transform back to a representation in terms of heat operators and define

$$
f_{\varepsilon}(s)=\operatorname{Tr}\left[\ln \varrho\left(e^{-s L_{\varepsilon}^{\dagger} L_{\varepsilon}}-e^{-s L_{\varepsilon} L_{\varepsilon}^{\dagger}}\right) \chi_{\lambda}\right]
$$

(3.16) can then be written

$$
\frac{\partial}{\partial \varepsilon} \ln \operatorname{det}_{\left\{z_{i}\right\}} L_{\varepsilon}^{\dagger} L_{\varepsilon}=\int_{0}^{\infty} d s \sum_{i=0}^{R} e_{i} z_{i} e^{-s z_{i}} f_{\varepsilon}(s)+O(1 / \lambda) .
$$

We have now to take the limit $z_{0} \rightarrow 0, z_{i} \rightarrow \infty, \lambda \rightarrow \infty, i>0$, subject to the conditions (2.17), which reduce in dimension 4 just to

$$
\begin{aligned}
& \sum_{i=1}^{R} e_{i} z_{i}=0 \\
& \sum_{i=1}^{R} e_{i} \ln z_{i}=-\ln z \\
& \sum_{i=1}^{R} e_{i} z_{i} \ln z_{i}=-z_{(1)} \ln z_{(1)} .
\end{aligned}
$$

The answer will be independent of $z_{(1)}$ as anticipated by Proposition 3. Assume at this point that $f_{\varepsilon}(s)$ has the asymptotic behavior, as $s \rightarrow 0$ :

$$
f_{\varepsilon}(s) \sim \frac{1}{s} c_{0}(\varepsilon)+c_{1}(\varepsilon)+c_{1+}(\varepsilon) \ln s+\ldots,
$$

where the remainder goes to zero as $s \rightarrow 0$. A discussion of this type of asymptotics for the heat kernels will be given in the appendix. (The logarithmic term is ordinarily not present for differential operators with smooth coefficients. It may appear, however, because of singularities in the coefficients. ${ }^{1}$ ) Given (3.18), for the

1 In the appendix we actually study $\int_{0}^{1} d \varepsilon f_{\varepsilon}(s)$, but this is actually all we need; see (3.22) below 
limit of (3.17) as $z_{i} \rightarrow 0, i>0$, and $\lambda \rightarrow \infty$ we find

$$
\begin{aligned}
\frac{\partial}{\partial \varepsilon} \ln \operatorname{det} L_{\varepsilon}^{\dagger} L_{\varepsilon}= & c_{0}(\varepsilon) z_{1} \ln z_{i}+\gamma c_{1+}(\varepsilon)-c_{1}(\varepsilon)+c_{1+}(\varepsilon) \ln z \\
& -\lim _{z \rightarrow 0}\left[\int_{0}^{\infty} d s \ln s f_{\varepsilon}^{\prime}\left(\frac{s}{z}\right) e^{-s}-z \int_{0}^{\infty} d s \ln s f_{\varepsilon}\left(\frac{s}{z}\right) e^{-s}\right],
\end{aligned}
$$

where $\gamma$ is Euler's constant $(\gamma=0.577 \ldots)$. In the absence of zero eigenvalue modes for the operators $L^{\dagger} L$ and $L L^{\dagger}$ the last term is zero. On the other hand, $L_{0}^{\dagger} L_{0}=-\partial^{2}$, so $\operatorname{det} L_{0}^{\dagger} L_{0}=1$. We summarize in

Proposition 4. Suppose that $L$ is of the form (3.2) where $1 / \varrho \partial_{\mu} \varrho$ is regular everywhere and $|\ln \varrho(x)|$ satisfies (3.11). Then

$$
\begin{aligned}
\ln \operatorname{det} L^{\dagger} L= & z_{(1)} \ln z_{(1)} \int_{0}^{1} d \varepsilon c_{0}(\varepsilon)+\ln z \int_{0}^{1} d \varepsilon c_{1+}(\varepsilon) \\
& +\int_{0}^{1} d \varepsilon\left[\gamma c_{1+}(\varepsilon)-c_{1}(\varepsilon)\right]
\end{aligned}
$$

where $c_{0}(\varepsilon), c_{1}(\varepsilon), c_{1+}(\varepsilon)$ are the asymptotic coefficients given by $(3.20), f_{\varepsilon}(s)$ is given by (3.17) with $\lambda \rightarrow \infty$ and $L_{\varepsilon}$ by (3.19). $z_{(1)}$ and $z$ are given by (3.19).

Since the coefficients of $L^{\dagger} L$ and $L L^{\dagger}$ are smooth, these asymptotic coefficients are straightforward to compute as is explained in Appendix A.

Returning to our example with $\varrho(x)$ given by (2.6) we note that $\varrho(x)>0$ and is regular everywhere except at a finite number of points $y_{i}$ where it behaves like

$$
\frac{\lambda_{i}^{2}}{\left|x-y_{i}\right|^{2}}+\text { smooth functions . }
$$

The analysis that led to Proposition 4 is still valid for $\varrho(x)$ given by (2.6). However, the singularities prevent us from computing the asymptotic coefficients in (3.20) in a straightforward manner. We can circumvent this difficulty by using our freedom in the choice of the operator $A_{0}$ in (2.20). This case is unique, because the leading order behavior of $\varrho(x)$ near a point of singularity agrees with that of a single instanton potential with the instanton position at that point. The determinant for the covariant Laplacian associated with the single instanton potential can be computed directly by spectral analysis [1]. In effect we shall calculate

$$
\ln \operatorname{det} L^{\dagger} L-\sum_{k=1}^{n} \ln \operatorname{det} L_{k}^{\dagger} L_{k}
$$

with $L_{k}$ given by (3.2) with $\varrho$ replaced by

$$
\varrho_{k}=1+\frac{\lambda_{k}^{2}}{\left|x-y_{k}\right|^{2}} \text {. }
$$

This allows us to go as far as is indicated by (3.20) in the asymptotics. The result is 
Proposition 5. Suppose that $L$ is as (3.2) with $\varrho$ given by (2.6). Then

$$
\begin{aligned}
\ln \operatorname{det} L^{\dagger} L= & -\frac{1}{16 \pi^{2}} z_{(1)} \ln z_{(1)} \int d x \ln \varrho \partial^{2} \ln \varrho \\
& +\frac{1}{96 \pi^{2}} \int d x\left\{\left[-\left(\partial^{2} \ln \varrho\right)^{2}+\frac{1}{2}\left((\partial \ln \varrho)^{2}\right)^{2}\right]\right. \\
& \left.-\sum_{k=1}^{n}\left[-\left(\partial^{2} \ln \varrho_{k}\right)^{2}+\frac{1}{2}\left(\left(\partial \ln \varrho_{k}\right)^{2}\right)^{2}\right]\right\} \\
& +\sum_{k=1}^{n} \ln \operatorname{det} L_{k}^{\dagger} L_{k}
\end{aligned}
$$

where

$$
\varrho_{k}(x)=1+\frac{\lambda_{k}^{2}}{\left|x-y_{k}\right|^{2}},
$$

the $\varrho(x)$ corresponding to a single instanton, and $L_{k}$ is given by (3.2) with $\varrho$ replaced by $\varrho_{k}$. Det $L_{k}^{\dagger} L_{k}$ is given by 't Hooft [1].

The proof is contained in the discussion of Appendix A.

We can express (3.25) in a form that agrees with the results of Brown and Creamer [4] using the property that

$$
(\partial \ln \varrho)^{2}+\partial^{2} \ln \varrho=0
$$

then we have

$$
\begin{aligned}
\ln \operatorname{det} L^{\dagger} L= & -\frac{1}{192 \pi^{2}} \int d x\left[\left(\partial^{2} \ln \varrho\right)^{2}-\sum_{k=1}^{n}\left(\partial^{2} \ln \varrho_{k}\right)^{2}\right] \\
& +\sum_{k=1}^{n} \ln \operatorname{det} L_{k}^{\dagger} L_{k} .
\end{aligned}
$$

\section{Appendix A: Asymptotics of the Heat Kernel}

We wish to discuss in this appendix the asymptotics of the heat kernel $e^{-s A}(x, x)$, where $A$ is a differential operator. The results have been stated and used in the main part of the paper. Proposition 1 describes the well-known classical results about differential operators with smooth coefficients. Proposition 4 relies on an extension of these results to operators with singular coefficients. It is this extension as given in Eq. (3.20), that we intend to discuss, but we unavoidably include an outline of the theory for operators with smooth coefficients.

For simplicity, we restrict the discussion to second order operators of the type that we use in this paper, i.e., operators on $H^{2}\left(\mathbb{R}^{4}\right)$ having $-\partial^{2}$ as the top order term.

We shall sketch the derivation of Proposition 1. At the same time we shall prove the following very restricted result about operators with singular coefficients. This result is essentially described in Eq. (3.20). At the end of the appendix we shall formulate a conjecture which generalizes Proposition A1. 
Proposition A1. Let $L$ be as in Proposition 1 and $g_{\varepsilon}(s)=\lim _{\lambda \rightarrow \infty} f_{\varepsilon}(s)$ with $f(s)$ as defined by (3.17). Then as $s \rightarrow 0$, we have an asymptotic expansion

$$
\int_{0}^{1} g_{\varepsilon}(s) d \varepsilon \sim \frac{1}{s} c_{0}+c_{1}+c_{1}+\ln s+\ldots,
$$

where

$$
\begin{aligned}
c_{0}= & -\frac{1}{16 \pi^{2}} \int d x \ln \varrho \cdot \partial^{2} \ln \varrho \\
c_{1}= & \frac{1}{96 \pi^{2}} \int \mathrm{dx}\left\{\left[-\left(\partial^{2} \ln \varrho\right)^{2}+\frac{1}{2}\left((\partial \ln \varrho)^{2}\right)^{2}\right]\right. \\
& \left.-\sum_{k=1}^{n}\left[-\left(\partial^{2} \ln \varrho_{k}\right)^{2}+\frac{1}{2}\left(\left(\partial \ln \varrho_{k}\right)^{2}\right)^{2}\right]\right\} \\
& +\frac{n \cdot \gamma}{12}-n \cdot\left(2 R-\frac{17}{72}-\frac{1}{6} \ln 2\right)-\sum_{k=1}^{n} \frac{1}{6} \ln \lambda_{k}, \quad c_{1+}=n / 12
\end{aligned}
$$

with

$$
R=\frac{1}{12}-\zeta^{\prime}(-1)=\frac{1}{12}(\ln 2 \pi+\gamma)+\frac{1}{2 \pi^{2}} \sum_{s=1}^{\infty} \frac{\ln s}{s^{2}}, \quad \gamma=0.577 \ldots
$$

Remarks. We emphasize that what is contained in this proposition is all we can prove about asymptotic expansions of heat kernels for operators with singular coefficients. Furthermore, the information in (A.1) is completely equivalent to the calculation of the determinant of an operator $L^{\dagger} L$ where $L$ is the general operator of the form assumed in Proposition A1. Proposition 5, which contains the results of this general calculation will in fact be demonstrated simultaneously with Proposition A1 in the proof that will be given below.

We also stress that (3.20), which is a stronger form of (A.1) will not be proved. It is rather a consequence of the conjecture formulated at the end of this appendix.

Proof of Propositions $A 1$ and 5. Proposition 4 shows that $c_{0}, c_{1}, c_{1+}$, as they appear in (A.1) are defined uniquely by the coefficients of $z_{(1)} \ln z_{(1)}, \ln z$ and the cutoff-independent term in $\ln \operatorname{det} L^{\dagger} L$, and vice versa. Thus the asymptotics of the heat kernels involved in (3.17) is known, if and only if the determinant is known. For the case of regular coefficients in $L$, it is simpler to compute these asymptotic coefficients and then use the result to compute the determinant. For the case of singular coefficients some special information about the determinant of particular operators is used to study the asymptotics of (3.17) and the latter is used in turn to compute the general determinant.

To make transparent how the $\ln s$-terms appear in (A.1), we first sketch the derivation of the asymptotics of $e^{-s A}(x, x)$ with the coefficients of $A$ regular. In part (b) below we discuss singular coefficients.

\section{a) Regular Coefficients}

Let

$$
A=-1 \cdot \partial^{2}+V_{\mu} \partial_{\mu}+V_{0},
$$


a second order differential operator: $\left[H^{2}\left(\mathbb{R}^{4}\right)\right]^{M} \rightarrow\left[L^{2}\left(\mathbb{R}^{4}\right)\right]^{M}$ where $M$ is the order of the $C^{\infty}$ matrices $V_{\mu}(x), V_{0}(x)$. A systematic way of studying $e^{-s A}(x, x)$ for small $s$ is by making use of the Levy expansion of the heat kernel in conjunction with a Taylor's expansion of the coefficients of the operator $A[10]$. Define the operators

$$
\begin{aligned}
& H_{0}=-1 \cdot \partial^{2} \\
& w=V_{\mu} \partial_{\mu}+V_{0} .
\end{aligned}
$$

The Levy expansion of the operator $e^{-s A}$ is given by the formal series

$$
\begin{aligned}
e^{-s A}= & \sum_{n=0}^{\infty}(-1)^{n} \int_{0}^{s} d r_{1} \int_{0}^{r_{1}} d r_{2} \ldots \int_{0}^{r_{n}-1} d r_{n} e^{-\left(s-r_{1}\right) H_{0}} w \\
& \cdot e^{-\left(r_{1}-r_{2}\right) H_{0}} w \ldots w e^{-r_{n} H_{0}}
\end{aligned}
$$

which is absolutely convergent if $A$ is a matrix, but only asymptotic if $A$ is a differential operator with smooth coefficients. Looking at the kernel of the integral operator $e^{-r H_{0}}$ we see that

$$
e^{-r H_{0}}(x, z)=e^{-r H_{0}}(x-z)=\frac{1}{r^{2}} e^{-H_{0}}\left(\frac{x-z}{\sqrt{r}}\right) .
$$

We then find that the $n$-th term in the expansion (A.5) evaluated on the diagonal is

$$
\begin{aligned}
\left(e^{-s A}\right)^{(n)}(x, x)= & s^{-n-2}(-1)^{n} \int_{0}^{1} d r_{1} \int_{0}^{r_{1}} d r_{2} \ldots \int_{0}^{r_{n}-1} d r_{n} \\
& \cdot \int \prod_{k=1}^{n}\left(d y_{k}\right) e^{-\left(1-r_{1}\right) H_{0}}\left(\frac{x-y_{1}}{\sqrt{s}}\right) w\left(y_{1}\right) \ldots w\left(y_{n}\right) e^{-r_{n} H_{0}}\left(\frac{y_{n}-x}{\sqrt{s}}\right) .
\end{aligned}
$$

We next make a change of variables and write $u_{j}=\left(x-y_{i}\right) / \sqrt{s}$ for $j=1$ to $n$. This allows us to write (A.6) as

$$
\begin{aligned}
\left(e^{-s A}\right)^{(n)}(x, x)= & s^{n-2}(-1)^{n} \int_{0}^{1} d r_{1} \int_{0}^{r_{1}} d r_{2} \ldots \int_{0}^{r_{n}-1} d r_{n} \\
& \cdot \int \prod_{k=1}^{n}\left(d u_{k}\right) e^{-\left(1-r_{1}\right) H_{0}}\left(u_{1}\right) w\left(x-\sqrt{s} u_{1}\right) \ldots w\left(x-\sqrt{s} u_{n}\right) \\
& \cdot e^{-r_{n} H_{0}}\left(-u_{n}\right) .
\end{aligned}
$$

In (A.7) we have

$$
w\left(x-\sqrt{s} u_{j}\right)=\frac{1}{\sqrt{s}} V_{\mu}\left(x-\sqrt{s} u_{j}\right) \frac{\partial}{\partial u_{j}}+V_{0}\left(x-\sqrt{s} u_{j}\right)
$$

Aside from the overall factor of $s^{n-2}$, the $s$-dependence of the expression on the right-hand side of (A.7) lies in the dependence of the functions $V_{\mu}$ and $V_{0}$ on the quantity $x-\sqrt{s} u_{j}$. These functions are by hypothesis $C^{\infty}$ functions on $\mathbb{R}^{4}$ so we can expand them in a Taylor series in $\sqrt{s}$ to any order. When these Taylor series are substituted in (A.7), one obtains the desired asymptotic expansion for $e^{-s A}$, as 
follows. When the series for the w's are multiplied out in (A.7) the coefficient of each power of $\sqrt{s}$ is a polynomial in the $u$ 's with coefficients formed by products of $w(x)$ and its derivatives. The $u$ 's then appear only in the exponential and in those polynomials. The $u$-integrations immediately yield then $\delta$-functions and derivatives of $\delta$-functions in the variables $r_{i}$ and the $r$-integrations become trivial to do. One observes that the coefficients of the odd powers of $\sqrt{s}$ are trivially zero. In this way it becomes apparent that the Levy expansion (A.5) is a systematic way of studying the asymptotics of $e^{-s A}(x, x)$ in the sense that the successive terms are of higher order in $s$, and that the $a_{k}(x)$ in Proposition 2 are polynomial functions of $V_{1}^{\mu}, V_{0}$ and their derivatives. Thus the latter Proposition is proved.

\section{b) Singular Coefficients}

The situation is more difficult when the coefficients are not $C^{\infty}$ functions on $\mathbb{R}^{4}$ but rather have singularities. In this case, the asymptotic expansion of the kernel $e^{-s A}(x, x)$ cannot be defined beyond a certain order in $s$ by using the method outlined above. Precisely, the coefficients $a_{k}(x)$ of Proposition 2, being polynomials in $V_{1}^{\mu}, V_{0}$ and their derivatives of an order increasing with $k$, eventually become arbitrarily singular at the points where $V_{1}^{\mu}, V_{0}$ are singular. Thus, for example, if we are trying to study the limit $s \rightarrow 0$ of a quantity like $\int e^{-s A}(x, x)(d x)$, we can only go as far as a certain order in $s$ by using the local asymptotics of Proposition 2, because the coefficients of the higher powers of $s$ are found to be infinite by this method. These infinities are interpreted as being the result of an illegitimate expansion in $s$, e.g., of a function like $1 / s, s^{1 / 2}$, or $\ln s$. Thus, the expansion is either to be rearranged to give the correct finite result or it may contain new types of terms, like $\ln s$ or fractional powers of $s$. This explains why an expansion of the type (A.1) may be expected.

Our aim here is to prove just the expansion (A.1). If $L_{\varepsilon}$ is as in Proposition 1, $L_{\varepsilon}^{\dagger} L_{\varepsilon}$ and $L_{\varepsilon} L_{\varepsilon}^{\dagger}$ are of the form (A.3) with

$$
\begin{aligned}
& L_{\varepsilon}^{\dagger} L_{\varepsilon}=-\partial^{2}+2 i \varepsilon \bar{\tau}_{\mu \nu} \varrho_{, \mu} \partial_{v}+\frac{\varepsilon^{2}}{4}(\partial \varrho)^{2}-\frac{\varepsilon}{2}\left(\partial^{2} \varrho\right) \\
& L_{\varepsilon} L_{\varepsilon}^{\dagger}=-\partial^{2}+2 i \varepsilon \tau_{\mu \nu} \varrho_{, \mu} \partial_{v}+\frac{\varepsilon^{2}}{4}(\partial \varrho)^{2}+\frac{\varepsilon}{2}\left(\partial^{2} \varrho\right),
\end{aligned}
$$

where $\bar{\tau}_{\mu v}$ is defined by (2.5) and

$$
\tau_{\mu v}=\frac{1}{4 i}\left(\beta^{\mu^{\dagger}} \beta^{v}-\beta^{v^{\dagger}} \beta^{\mu}\right)
$$

All the dependence of $L_{\varepsilon}^{\dagger} L_{\varepsilon}$ and $L_{\varepsilon} L_{\varepsilon}^{\dagger}$ on $\varepsilon$ is shown explicitly in (A.8). Near the singular points the $\varrho$ in (A.8) is assumed to have the same behavior as the $\varrho$ given in (2.6). Thus, the coefficient of $\partial_{v}$ in either expression in (A.8) behaves like $\left(x-y_{k}\right) /\left|x-y_{k}\right|^{2}$ and the zero-order terms like $1 /\left|x-y_{k}\right|^{2}$.

Recall that we wish to study the asymptotics (A.1) through logarithmic order. Now the logarithmic term has the same dimensions as the constant term. So suppose we attempt to carry the asymptotics to constant order by using the same analysis as we applied to the case of regular coefficients above. Then we notice that 
we obtain for the constant term an integral which diverges logarithmically at the points $y_{k}$. For the heat kernel has dimensions (length) ${ }^{-1}$, the parameter $s$ has dimensions (length) ${ }^{-2}$ and, therefore, the coefficient $a_{0}(x)$ of $s^{0}$ must have dimensions (length) ${ }^{-4}$; and since $\varepsilon$ is dimensionless in (A.8) that implies that $a_{0}(x)$ must behave like $1 /\left|x-y_{k}\right|^{4}$.

The idea is now to subtract $a$ quantity with the same leading order behavior so that the logarithmic divergence disappears. Propositions 4 establishes a correspondence between the asymptotics (A.1) through logarithmic order and the determinant, so we can study the corresponding subtraction on the determinant. Thus, consider the operators

$$
L_{k, \varepsilon}=\varrho_{k}^{-\varepsilon / 2} L_{0} \varrho_{k}^{\varepsilon / 2}
$$

where

$$
\varrho_{k}=1+\frac{\lambda_{k}^{2}}{\left|x-y_{k}\right|^{2}} .
$$

Near $x=y_{k}, \varrho_{k}$ has the same leading order behavior as (2.6). Write $L_{k}$ simply for $L_{k 1} . L_{k}^{\dagger} L_{k}$ is the Laplacian of isospin $-1 / 2$ scalars in the background of a single instanton of size $\lambda_{k}$ at $y_{k}$ and $\operatorname{det} L_{k}^{\dagger} L_{k}$ is computed by 't Hooft [1]. We now write the determinant

$$
\begin{aligned}
\ln \operatorname{det} L^{\dagger} L= & \left(\ln \operatorname{det} L^{\dagger} L-\sum_{k=1}^{n} \ln \operatorname{det} L_{k}^{\dagger} L_{k}\right) \\
& +\sum_{k=1}^{n} \ln \operatorname{det} L_{k}^{\dagger} L_{k} .
\end{aligned}
$$

According to 't Hooft (see [1], p. 3441, Eq. (7.6) with $\mu_{0}^{2}=z$ )

$$
\ln \operatorname{det} L_{k}^{\dagger} L_{k}=\frac{1}{12} \ln z+2 R-\frac{1}{6} \ln 2-\frac{17}{72}
$$

while by Proposition 4,

$$
\ln \operatorname{det} L^{\dagger} L-\sum_{k=1}^{n} \ln \operatorname{det} L_{k}^{\dagger} L_{k}=z_{(1)} \ln z_{(1)} d_{0}-d_{1},
$$

where $d_{0}$ and $d_{1}$ are obtained from the asymptotic expansion

$$
\begin{aligned}
\int_{0}^{1} d \varepsilon & \operatorname{tr}\left[\frac{\varrho}{2} \operatorname{Tr}\left(e^{-s L_{\varepsilon}^{\dagger} L_{\varepsilon}}-e^{-s L_{\varepsilon}^{\dagger} L_{\varepsilon}^{\dagger}}\right)-\sum_{k=1}^{n} \frac{\varrho_{k}}{2} \operatorname{Tr}\left(e^{-s L_{k \varepsilon}^{\dagger} L_{k \varepsilon}}-e^{-s L_{k \varepsilon} L_{k \varepsilon}^{\dagger}}\right)\right] \\
& \sim \frac{1}{S} d_{0}+d_{1}+\ldots
\end{aligned}
$$

We now show that (A.14) is valid to the indicated order and that

$$
\begin{aligned}
d_{0}= & \frac{1}{16 \pi^{2}} \int d x \ln \varrho \cdot \partial^{2} \ln \varrho \\
d_{1}= & -\frac{1}{96 \pi^{2}} \int d x\left\{\left[-\left(\partial^{2} \ln \varrho\right)^{2}+\frac{1}{2}\left((\partial \ln \varrho)^{2}\right)^{2}\right]\right. \\
& \left.-\sum_{k=1}^{n}\left[-\left(\partial^{2} \ln \varrho_{k}\right)^{2}+\frac{1}{2}\left(\left(\partial \ln \varrho_{k}\right)^{2}\right)^{2}\right]\right\} .
\end{aligned}
$$


We claim that (A.14) can be obtained using the Levy expansion and Taylor series as described above in the case of regular coefficients. To see how this procedure works, consider the first term in the Levy expansion for (A.14). It is of the form

$$
\begin{aligned}
& -\frac{1}{S} \int(d x)\left[\frac{\varrho(x)}{2} \int_{0}^{1} d v \int(d y) e^{-(1-r) H_{0}}(y) v(x-\sqrt{s} y) e^{-r H_{0}}(y)\right. \\
& \left.+\frac{1}{S} \sum_{k=1}^{n} \frac{\varrho_{k}(x)}{2} \int_{0}^{1} d r \int(d y) e^{-(1-r) H_{0}}(y) v_{k}(x-\sqrt{s} y) e^{-r H_{0}}(y)\right] .
\end{aligned}
$$

The functions $v(x), v_{k}(x)$ are combinations of coefficients in $L^{\dagger} L, L L^{\beta}, L_{k}^{\beta} L_{k}, L_{k} L_{k}^{\dagger}$ and they agree to leading order near $x=y_{k}$. We now rewrite (A.15) in the form:

$$
\begin{aligned}
& -\frac{1}{s} \int(d x)\left[\frac{\varrho(x)}{2} \int_{0}^{1} d r(d y) e^{-(1-r) H_{0}}(y)\left(v(x-\sqrt{s} y)-\sum_{k=1}^{n} v_{k}(x-\sqrt{s} y)\right) e^{-r H_{0}}(y)\right. \\
& \left.\quad+\left(\sum_{k=1}^{n} \frac{\varrho_{k}(x)}{2}-\frac{\varrho(x)}{2}\right) \int_{0}^{1} d r \int(d y) e^{-(1-r) H_{0}}(y) v_{0}(x-\sqrt{s} y) e^{-r H_{0}}(y)\right]
\end{aligned}
$$

Because of the subtractions, $v(x-\sqrt{s} y)-\sum_{k=1}^{n} v_{k}(x-\sqrt{s} y)$ becomes a regular quantity, in the first term, while in the second $v_{0}(x-\sqrt{s} y)$ can be expanded to a higher order than without the subtractions, without making the $x$-integrations divergent.

For the higher terms in the Levy expansion for (A.14) (which are necessary in order to prove the indicated asymptotic expansion), the subtractions allow us to carry the Taylor series in $\sqrt{s}$ for the $w$ 's in the generic expression (A.7) one order higher than without them. This allows us to calculate the coefficients of $s^{-1}$ and $s^{0}$ and with a certain amount of algebra one can obtain the formulas (A.15).

(A.12) and (A.13) combined in (A.11) give us

$$
\ln \operatorname{det} L^{\dagger} L=z_{(1)} \ln z_{(1)} d_{0}+\frac{n}{12} \cdot \ln z-d_{1}+n \cdot\left(2 R-\frac{1}{6} \ln 2-\frac{17}{72}\right) .
$$

By Proposition 4 we obtain the coefficients in (A.1):

$$
\begin{aligned}
& c_{0}=d_{0} \\
& c_{1}=\gamma \cdot \frac{n}{12}-d_{1}-n \cdot\left(2 R-\frac{1}{6} \ln 2-\frac{17}{72}\right) \\
& c_{1+}=\frac{n}{12} .
\end{aligned}
$$

One would like to be able to prove an asymptotic expansion of the form (3.20) directly and also have a systematic way of computing asymptotic coefficients to arbitrary order. The problem basically amounts to studying $e^{-s H}$ where $H$ is a differential operator with singular coefficients. One can see that although the naive local asymptotic expansion gives very singular results, the kernel itself is much less singular at the singularities of the coefficients, so that in general one can define quantities like $\int \operatorname{tr} e^{-s H}(x, x) d x$ or $\int \chi(x) e^{-s H}(x, x) d x$ where $\chi(x)$ is in the Schwartz 
space or else has a finite number of sufficiently mild singularities. Thus, there are also different ways in which one can study the asymptotics of $e^{-s H}$. One may, for example consider the global quantity $\operatorname{Tr} e^{-s H}$; or more generally the local asymptotics of $e^{-s H}(x, x)$ in the sense of distributions; or again the asymptotics in the sense of distributions of $\chi(x) e^{-s H}(x, x)$ where $\chi(x)$ is singular at the points of singularity of the coefficients of $H$. The typical kind of result that we expect is exemplified in:

\section{Conjecture}

Let $H$ be a second order self-adjoint differential operator on $\mathbb{R}^{n}$ of the form $-\partial^{2}+V_{1}^{i}(x) \partial_{i}+v_{0}(x)$, where $V_{1}^{i}(x), V_{0}(x)$ are regular everywhere except at a finite number of points $y_{k}$ where they are allowed to have singularities of the form $1 /\left|x-y_{k}\right|^{\alpha}, \alpha<1$ and $1 /\left|x-y_{k}\right|^{\beta}, \beta<2$, respectively. Then $e^{-s H}(x, x)$ has an asymptotic expansion in the sense of distributions of the form

$$
\sum_{k} \sum^{-n / 2} a_{k}(x) s^{k}+\sum_{k} \sum_{\substack{m, j=0 \\ L=\left(L_{1} \ldots L_{n}\right)}}^{\infty} A_{m j L}^{(k)} S^{\alpha_{m}}|\ln s|^{\beta_{j}} \delta^{(L)}\left(x-y_{k}\right),
$$

where $a_{k}(x)$ are local functions of the nonsingular parts of $V_{0}(x), V_{1}^{i}(x), \alpha_{m}, \beta_{j}$ are increasing sequences of exponents, $A_{m j L}^{k}$ are constants and $\delta^{L}\left(x-y_{k}\right)$ is the derivative $\left.\partial\right|^{L} \mid / \partial x_{1}^{L_{1}} \ldots \partial x_{n}^{L_{n}} \delta\left(x-y_{k}\right)$ of the Dirac $\delta$-function.

\section{Operators with SU(2) Yang-Mills Background Field}

We now derive the expressions (2.27) for the $\beta$ and $\gamma$ of Proposition 2, i.e., consider the asymptotics of the heat kernel, $\exp \left(-t \Delta_{A}\right)$ with $\Delta_{A}$ defined as follows. Let $E$ be the two-dimensional complex vector bundle over $\mathbb{R}^{4}$. Let $A$ be a $C^{\infty} \mathrm{SU}(2)$ connection on $E$. Require that the Lie algebra valued 2 -form, $F=d A+1 / 2[A, A]$ has finite Yang-Mill's action; which is to say that $\operatorname{Tr} \int_{\mathbb{R}^{4}} F \wedge{ }^{*} F$ is finite. The connection $A$, defines a covariant derivative, $D_{A}: \Omega^{0}\left(\mathbb{R}^{4}, E\right) \rightarrow \Omega^{1}\left(\mathbb{R}^{4}, E\right)$; a first order differential operator which maps $C^{\infty}$ sections of $E$ into $C^{\infty} 1$-forms with values in $E$. $E$ has a natural Hermitian metric defined on each fiber. Define $\mathscr{S}(E) \rightarrow \Omega^{0}\left(\mathbb{R}^{4}, E\right)$ to be the set of sections whose components with respect to any orthonormal frame generating $E$ are elements of the Schwartz space $\mathscr{S}\left(\mathbb{R}^{4}\right)$. The inner product on $\mathscr{S}(E)$ is defined to be $\left\langle s, s^{\prime}\right\rangle=\int_{\mathbb{R}^{4}} d^{4} x\left(s, s^{\prime}\right)(x)$. With respect to this inner product $D_{A}$ has an adjoint $D_{A}^{*}$. We form the covariant Laplacian, $\Delta_{A}=D_{A} D_{A}^{*}$ $+D_{A}^{*} D_{A}$; a second order elliptic operator from $\mathscr{S}(E)$ to $\mathscr{S}(E)$. With respect to some orthonormal frame for $E$,

$$
\Delta_{A}=-\partial^{2} \otimes 1-\frac{i}{2}\left(\partial_{\mu} A_{\mu}^{k}+2 A_{\mu}^{k} \partial_{\mu}\right) \otimes \sigma^{k}+\frac{1}{4} A_{\mu}^{k} A_{\mu}^{k} \otimes 1,
$$

where the $\sigma^{k}$ are the Pauli matrices.

Define the $n$ 'th asymptotic function by the formula:

$$
a_{n}(x)=\left.\frac{1}{(n+2) !} \frac{\partial^{(n+2)}}{\partial s^{(n+2)}}\left(s^{2} \operatorname{tr}\left(e^{-s \Delta_{A}}-e^{-s \Delta_{0}}\right)(x, x)\right)\right|_{s=0},
$$


where

$$
\Delta_{0}=-\partial^{2} \otimes 1, \quad n=-1,0,1, \ldots
$$

From the definition it is clear that each asymptotic function defines a function on $\mathbb{R}^{4}$ [i.e., $a_{n}(x)$ is gauge invariant]. Because the coefficients of $\Delta_{A}$ are by hypothesis $C^{\infty}$, it is straightforward to compute its asymptotic functions (see Appendix A). For the operator $\Delta_{A}$ above we find that

$$
\operatorname{tr}\left(e^{-s \Delta_{A}}-e^{-s \Delta_{0}}\right)(x, x)=+\frac{1}{16 \pi^{2}} \cdot \frac{1}{24} \cdot 2 \operatorname{Tr}\left(F_{\mu \nu} F_{\mu \nu}\right)(x)+O(s) .
$$

For self or anti-self dual connections on $\mathbb{R}^{4}$, the asymptotic coefficient, $a_{0}$, defined by

$$
\begin{aligned}
a_{0} & =\int d^{4} x \lim _{s \rightarrow 0} \frac{\partial^{2}}{\partial s^{2}} \frac{1}{2} s^{2} \operatorname{Tr}\left(e^{-s \Delta_{A}}-e^{-s \Delta_{0}}\right)(x, x) \\
& =\int d^{4} x a^{0}(x)
\end{aligned}
$$

is proportional to the winding (instanton) number of the connection.

\section{Appendix B: Trace Formulas for Heat Kernels}

In this appendix we show how the quantity

$$
\operatorname{Tr}\left(e^{-s D D^{\dagger}}-e^{-s D^{\dagger}} D\right)
$$

can be computed explicitly as a function of $s$ for $D$ an elliptic differential operator on some open manifold with $L^{2}$ boundary conditions. This expression appears in Sect. IIc). We note first that if $D$ were an elliptic differential operator on a compact manifold, then

$$
\operatorname{Tr}\left(e^{-s D D^{\dagger}}-e^{-s D^{\dagger} D}\right)=\operatorname{Tr}\left(\Pi_{+}-\Pi_{-}\right),
$$

where $\Pi_{+}\left(\Pi_{-}\right)$is the projection onto the zero-eigenspace of $D D^{\dagger}\left(D^{\dagger} D\right)$. The argument is simple: The spectra of both $D D^{\dagger}$ and $D^{\dagger} D$ are discrete and the eigenvalues behave like some power of the eigenvalue number. Thus the trace of each term is finite. On the other hand $D^{\dagger} D$ and $D D^{\dagger}$ have the same eigenvalues, except perhaps for 0 , and their eigenspaces are isomorphic, for if $\lambda \neq 0$ is an eigenvalue of $D^{\dagger} D$ so that $D^{\dagger} D \psi=\lambda \psi$ for some $\psi$, then $\left(D D^{\dagger}\right)(D \psi)=\lambda(D \psi)$.

Now an elliptic operator on an open manifold may have a continuous spectrum, so the above arguments do not apply. All we know is that if $D$ is Fredholm,

$$
\lim _{s \rightarrow \infty} \operatorname{Tr}\left(e^{-s D D^{\dagger}}-e^{-s D^{\dagger} D}\right)=\operatorname{Tr}\left(\Pi_{+}-\Pi_{-}\right)
$$

and, even if $D$ is not Fredholm, we can straightforwardly compute the asymptotic behavior of (B.1) as $s \rightarrow 0$. These two different limits can be used as boundary conditions, for as we will explain, the following proposition can be used to calculate the derivative of (B.1) with respect to $s$ explicitly as a function of $s$ [11]. 
Proposition B1. Let $L$ be a first order elliptic differential operator on the Hilbert space $K=\bigoplus_{1}^{M} L^{2}\left(\mathbb{R}^{n}\right)$ :

$$
D=i \delta^{j}(x) \partial_{j}+i \Phi(x),
$$

where $\delta^{i}(x), \Phi(x)$ are $M \times M$ matrices of smooth functions. Let $\delta^{i}$, $\Phi$ denote the multiplication operators by $\delta^{i}(x), \Phi(x)$. Suppose $z \notin \mathbb{R}_{+}$and let $\left(D D^{\dagger}+z\right)^{-1}(x, y)$, $\left(D^{\dagger} D+z\right)^{-1}(y, x)$ denote the kernel functions of the indicated integral operators. Then

$$
\begin{gathered}
2 z \operatorname{tr}\left[\left(D^{\dagger} D+z\right)^{-1}-\left(D D^{\dagger}+z\right)^{-1}\right](x, y) \\
=\left(\frac{\partial}{\partial x^{i}}+\frac{\partial}{\partial y^{i}}\right) J_{z}^{i}(x, y)+A_{z}(x, y),
\end{gathered}
$$

where $J_{z}^{i}(x, y)$ is the kernel of the integral operator

$$
J_{z}^{i}(x, y)=i \operatorname{Tr}\left(D\left(D^{\dagger} D+z\right)^{-1} \delta^{i \dagger}\right)(x, y)-i \operatorname{Tr}\left(D^{\dagger}\left(D D^{\dagger}+z\right)^{-1} \delta^{i}\right)(x, y)
$$

and $A_{z}(x, y)$ is the kernel of an integral operator on $L^{2}\left(\mathbb{R}^{n}\right)$ that can be represented as the trace of commutators of multiplication operators by smooth functions and the integral operator $D\left(D^{\dagger} D+z\right)^{-1}$ and $D^{\dagger}\left(D D^{\dagger}+z\right)^{-1}[12]$.

We remark that the above result can be extended to differential operators of arbitrary order as well as to arbitrary manifolds.

To apply this proposition to (B.1) we take the inverse Laplace transform of (B.5), as follows:

$$
\begin{aligned}
2 \int_{C} \frac{d z}{2 \pi i} e^{-s z}\left(\frac{1}{z-D D^{\dagger}}-\frac{1}{z-D^{\dagger} D}\right)(x, y)= & \left(\frac{\partial}{\partial x^{i}}+\frac{\partial}{\partial y^{i}}\right) \int_{C} \frac{d z}{2 \pi i} e^{-s z} J_{-z}^{i}(x, y) \\
& +\int_{C} \frac{d z}{2 \pi i} e^{-s z} A_{-z}(x, y),
\end{aligned}
$$

where $C$ is the contour shown in Fig. 1. The contour integration can be done immediately:

$$
\int_{C} \frac{d z}{2 \pi i} e^{-s z} \frac{1}{z-D D^{\dagger}}=e^{-s D D^{\dagger}}
$$

Now the kernels of the heat operators are smooth, so we can evaluate on the diagonal, $y=x$. It is then easy to see that the $A_{z}$-term in (B.5) gives no contribution. The result is

$$
\begin{aligned}
& -\frac{\partial}{\partial s} \operatorname{Tr}\left(e^{-s D D^{\dagger}}-e^{-s D^{\dagger}} D\right)(x, x) \\
& =\frac{\partial}{\partial x^{i}} i \operatorname{Tr}\left[D e^{-s D^{\dagger} D} \delta^{i \dagger}-D^{\dagger} e^{-s D D^{\dagger}} \delta^{i}\right](x, x) .
\end{aligned}
$$

Now we integrate over all space and find that the derivative of (B.1) with respect to $s$ is given by a surface integral at infinity:

$$
\lim _{R \rightarrow \infty} \int_{S_{R}^{n-1}} d s_{i} i \operatorname{Tr}\left[D e^{-s D^{\dagger} D} \delta^{i \dagger}-D^{\dagger} e^{-s D D^{\dagger}} \delta^{i}\right](x, x) .
$$




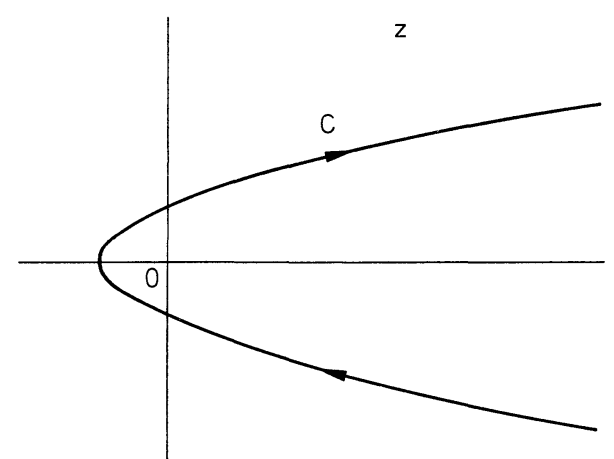

Fig. 1. Contour of integration for the definition of $e^{-t H}$

An asymptotic expansion of the integrand as $|x| \rightarrow \infty$ is done systematically by using the expansion of the heat kernel around the highest order part (for details, see [10], end of Sect. 3). Carrying out the calculation we find that the result is zero. Thus (B.1) is a constant. The constant can be computed by evaluating the limit as $s \rightarrow 0$, according to the standard methods described in Appendix A. We find

$$
\operatorname{Tr}\left[e^{-s D D^{\dagger}}-e^{-s D^{\dagger} D}\right]=-\frac{1}{32 \pi^{2}} \int \operatorname{Tr}\left({ }^{*} F(x) \cdot F(x)\right) d x .
$$

But now it is known that the index of $D$, which is the same as $\operatorname{Tr}\left(\Pi_{-}-\Pi_{+}\right)$is equal to the negative of this expression. This proves the formula at the end of Sect. 2.

\section{Appendix C: Alternative Methods of Regularization}

In this appendix we note the relation of our regularization scheme to others that are popular in the literature. In the analytic or $\zeta$-function scheme, one defines

$$
D(\lambda)=-\frac{1}{\Gamma(2-\lambda)} \int_{0}^{\infty} \frac{d s}{s^{\lambda}} \operatorname{Tr}\left(e^{-s A}-e^{-s A_{0}}\right)
$$

for given operators $A, A_{0}$ and $\operatorname{Re} \lambda \ll 0$. The ratio of the determinants of $A$ and $A_{0}$ is then defined as the finite part of the analytic continuation of $D(\lambda)$ to $\lambda=1$. To see how this works consider a second order differential operator on $\mathbb{R}^{4}$ as in Sect. 2. Let

$$
f(s)=s \operatorname{Tr}\left(e^{-s A}-e^{-s A_{0}}\right)
$$

a regular function of $s$. We can integrate by parts in the formula for $D(\lambda)$ to get for $\operatorname{Re} \lambda \ll 0$

$$
D(\lambda)=-\frac{1}{\lambda(\lambda-1) \Gamma(2-\lambda)} \int_{0}^{\infty} \frac{d s}{s^{\lambda-1}} f^{\prime \prime}(s) .
$$


As $\lambda \rightarrow 1$ this expression can be evaluated immediately:

$$
D(\lambda) \rightarrow-\frac{f^{\prime}(0)}{\lambda-1}-f^{\prime}(0)(1+\gamma)-\int_{0}^{\infty} d s \ln s f^{\prime \prime}(s) .
$$

The finite part of this is seen to be

$$
\operatorname{det} A / A_{0}=-\int_{0}^{\infty} d s \cdot \ln s f^{\prime \prime}(s)-f^{\prime}(0)(1+\gamma),
$$

where $\gamma=0.577$ is Euler's constant. This is immediately seen to be the term $\alpha$ in Proposition 2b.

Similar remarks apply to a definition of the determinant as the finite part of the integral

$$
\int_{\varepsilon}^{\infty} \frac{d s}{s} \operatorname{Tr}\left(e^{-s A}-e^{-s A_{0}}\right)
$$

as $\varepsilon \rightarrow 0$.

Note that each of the two definitions given in this appendix is unambiguous. For the significance of the dependence of the determinant on the parameter $z$, the reader is referred to 't Hooft's paper [1] for details

Acknowledgements. We would like to thank R. Bott, R. Jackiw, and A. Jaffe for conversation and encouragement.

\section{References}

1. 't Hooft, G.: Phys. Rev. D 14, 3432 (1976)

2. Jackiw, R., Nohl, C., Rebbi, C.: Phys. Rev. D 15, 1642 (1977)

3. Schwarz, A.S. : Lett. Math. Phys. 2, 201-205 (1978); ibid. 247-252; Commun. Math. Phys. 64, 233-268 (1979)

4. Brown, L.S., Creamer, D.: Phys. Rev. D 18, 3695 (1978)

5. Jackiw, R., Rebbi, C.: Phys. Rev. D 16, 1052 (1977)

6. Bjorken, J.D., Drell, S.D.: Relativistic quantum mechanics, p. 152. New York: McGraw Hill 1964

7. Brown, L.S., Carlitz, R.D., Creamer, D.B., Lee, C.: Phys. Rev. D 17, 1583 (1978)

8. Goldberg, S.: Unbounded linear operators. New York: McGraw Hill 1966

9. Reed, M., Simon, B.: Fourier analysis and self-adjointness. New York: Academic Press 1975

10. Gilkey, P.: The index theorem and the heat equation. Boston: Publish or Perish 1974

11. Jackiw, R., Rebbi, C.: Phys. Rev. D 14, 517 (1976)

12. Callias, C.: Commun. Math. Phys. 62, 213-234 (1978)

Communicated by A. Jaffe

Received May 25, 1979; in revised form November 14, 1979 